\title{
An Antibiotic Protocol to Minimize Emergence of Drug-resistant Tuberculosis
}

\author{
Aquino L. de Espíndola ${ }^{1}$, Daniel Girardi ${ }^{1,2}$, T. J. P. Penna ${ }^{1,2}$, \\ Chris T. Bauch ${ }^{3}$, Brenno C. Troca Cabella ${ }^{4,5}$, Alexandre Souto Martinez ${ }^{2,4}$
}

1 Departamento de Fúsica, Instituto de Ciências Exatas - ICEx, Universidade Federal Fluminense, Rua Des. Ellis Hermydio Figueira, 783, 27.213-145, Volta Redonda, Rio de Janeiro, Brazil, aquinoespindola@id.uff.br,

2 National Institute of Science and Technology for Complex Systems - Brazil

3 Department of Applied Mathematics, University of Waterloo, 200 University Avenue West, Waterloo, ON, N2L 3G1, Canada,

${ }^{4}$ Faculdade de Filosofia, Ciências e Letras de Ribeirão Preto, Universidade de São Paulo, Avenida dos Bandeirantes, 3900, 14.040-901, Ribeirão Preto, São Paulo, Brazil,

5 SAPRA Assessoria S/S ltda - R.: Cid Silva César, 600, 13562-400 - São Carlos

- São Paulo, Brazil

Keywords: Complex Systems; Within-host Model; Epidemiology; Tuberculosis. 


\section{Introduction}

Tuberculosis (TB) is a world-wide [1] problem and it is estimated that one-third of the world's population is infected with the $M y$ cobacterium tuberculosis [2]. It is also the leading cause of death due to a single infectious disease [3]. TB is an airborne disease and can be transmitted from one person to another by cough, sneeze, speak etc. $[4,5]$.

Many mathematical models have been created to describe the dynamics of this illness and many others [6,7]. Most of these models deal with the problem of transmission dynamics with emergence of drug resistance [8-15], impact of other infections on TB $[16,17]$ and the role of dormancy in the persistence of the infection [18]. In contrast, within-host models have received little attention $[15,18$ 21].

Within-host models have several advantages for study the evolution and spread of a disease [22-26]. One of these advantages is that the host's health state is defined according to his/her internal population of pathogens. In transmission models, transitions among states are fixed parameters determined only by external factors. In contrast, in within-host models, transitions 
among host's health states emerge naturally from changes in the host's pathogen populations.

The possibility of defining a different set of parameters for each host is another important feature of these kinds of models. Immune response, initial load of pathogens, bacterial rate of dormancy and others parameters allow the creation of heterogeneous populations $[18,27-31]$. Within-host heterogeneity parameters (governed by the individual's biological characteristics) are useful to study TB spread because it is possible to simulate a large spectrum of virtual populations.

In principle, models of disease transmission from one individual to another can also be augmented by combining them with withinhost models. Contagion no longer occurs because of some fixed probability, but it also depends on the amount of pathogens involved in the process. Thus, the transmission of the disease among individuals on a population can be informed by monitoring host parameters.

In previous models, resistant strains [15] and bacterial dormancy [18] are treated separately. We propose to combine both effects simultaneously in a within-host model. Further, in our model, there is 
an interplay among sensitive/resistant pathogens, immune system cells, bacterial dormancy and antibiotics as the key features of the dynamics. Additionally, the immune system is assumed to depend on T-cell migration from the thymus, with a limited reproduction cycle.

The emergence of drug resistance due to the use of antibiotics [3234] is analyzed using three different treatment protocols. The standard, intermittent and oscillating intermittent protocols are characterized by the antibiotic doses and their periodicity. Outcomes are obtained for the within-host system with different Tcell migration rates and pathogen dormancy rates. Numerical calculations of the within-host model indicate the oscillating intermittent use of antibiotics as the most suitable protocol. It increases the susceptible number of individuals, but the number of drug resistant individuals is small.

This paper is organized as follows. In Section The Model, we describe the methods to build the within-host model of TB describing variables and parameters of the system. Also the dynamics of the within-host model is explained in details using coupled ordinary differential equations. Model numerical solutions and results are discussed in Sec. Results. In this section we present the way 
outcomes of the within-host dynamics are related to the host health states. Concluding remarks and our perspective on future studies are discussed in the last section.

\section{The Model}

Based on the models from Refs. [15,18], we propose a within-host model of Tuberculosis. We consider two types of M. tuberculosis strains: sensitive type, $S$, which can be killed by treatment with antibiotics; and a resistant type, $R$, which is resistant to the treatment. The influence of bacterial dormancy in the disease prevalence is also considered including dormant sensitive and dormant resistant types of bacteria, $S_{d}$ and $R_{d}$, respectively. We also model the emergence of drug resistance due the treatment with antibiotics.

The dynamics of bacterial populations and the immune system are modeled by using differential equations. The within-host system, defined by these set of equations, is solved numerically. We note that outcomes presented in the following sections are for one host only; the process of contagion or any interaction among hosts are not considered in this work. 
As mentioned above, the host health states are a consequence of the within-host dynamics. These states indicate the stage of the disease in which a host may be. In the case of TB, we define the possible health states for a host as: $X$, susceptible, $L_{i}$, latent; or $T_{i}$, infectious. The subscript $i=S, R$ defines the type of pathogen: sensitive or resistant to antibiotics, respectively.

Susceptible individuals, $X$, are those that had no contact with TB pathogens. They are healthy and their system is free of tuberculosis pathogens. Individuals previously susceptible who acquire TB pathogens may enter into a latency period. A latent state, $L_{i}$, is the stage when there are no disease symptoms. Finally, individuals in the infectious state, $T_{i}$, are in the active tuberculosis stage, i.e., the host is sick. Antibiotic treatment is applied in this stage of the disease.

M. Tuberculosis may enter mononuclear cells like the immune system cells, the T-cells [18]. A fraction of these bacteria go into a dormancy state for some time and consequently they do not reproduce [35]. During the dormancy state these pathogens are not detected by the immune system and they also can not be affected by the antibiotics $[18,36,37]$. Dormant sensitive and resistant bacteria will be represented as $S_{d}$ and $R_{d}$, respectively. 
On one hand, population of active sensitive pathogens, $S$, reproduce at rate $(1-q) \nu$ and they also may be converted to a dormant state, $S$ to $S_{d}$, at a rate $f$. On the other hand, the conversion back from the dormant to the active state, $S_{d}$ to $S$, occurs at a rate $g$. The $S$ type pathogens can be killed by the immune system response, $I$, or by the action of antibiotics with a clearance rate $\alpha$. The two types of strains compete for resources, thus a competition term [15] $\nu(S+R) S / k_{b}$ is added to the dynamics. This competition is only by their intrinsic growth rate and efficiency in utilizing available nutrients [38]. Then, as modeled in Ref. [15], a logistic competition term mimics the competition of survival between $S$ and $R$ pathogens.

Due to mutations, type $S$ pathogens may give rise to active resistant type bacteria, $R$, at rate $q \nu$. Reproduction of $R$ population that already exist occurs at rate $\nu_{1}$, which is lower than type $S$ because of an evolutionary cost [39]. Conversion from active state to a dormant state, $R$ to $R_{d}$ is also possible, as well as the conversion back to activity. Rates of conversion from active to dormant and dormant to active states are $f$ and $g$, respectively, as for sensitive pathogen.

Because of resistance to antibiotics, drug efficacy to clear $R$ type 
bacteria is reduced by a factor $\delta$. Consequently, the clearance rate due the use of antibiotics is lower than the one obtained to treat $S$ type infections. Resistant strains can be killed by the immune system similarly as the type $S$ pathogens. Analogously to $S$ strains, resistant bacteria has a competition term added to their dynamics, $\omega \nu(S+R) R / k_{b}$.

The within-host system evolves according to the equations:

$$
\begin{aligned}
\frac{d S}{d t} & =[(1-q) \nu-(f+\alpha)] S-\gamma S I+g S_{d}-\nu \frac{(S+R)}{k_{b}} S, \\
\frac{d S_{d}}{d t} & =f S-g S_{d}, \\
\frac{d R}{d t} & =q \nu S+\left[\nu_{1}-(f+\delta \alpha)\right] R-\gamma R I+g R_{d}-\omega \nu \frac{(S+R)}{k_{b}}(\mathbb{B},) \\
\frac{d R_{d}}{d t} & =f R-g R_{d} .
\end{aligned}
$$

Definition of the parameters used in Eqs. (1-4) as well as their respective ranges are in Table 1.

The last terms in Eqs. (1) and (3) are the competition terms between $S$ and $R$ strains. The amount of pathogens, $\Omega=S+R$, is the bacterial load inside the host's lung which determines his/her health condition. Mutations have a fitness cost [39] that may affect the reproduction ability of these strains [40].

The immune system, $I$, is modeled similarly to the dynamics pre- 
sented in Ref. [18]. Firstly, consider the thymus, an organ where immature T-cells stay until they fully mature $[41,42]$. Let $a(\Omega)$ be the immigration rate of T-cells from thymus, which depends on the pathogen density, given by:

$$
a(\Omega)=a_{1}-\frac{a_{1} \Omega^{m}}{a_{2}^{m}+\Omega^{m}},
$$

where $a_{1}$ is the recruitment rate of T-cells from thymus; $a_{2}$, pathogen load at which the immigration rate $a(\Omega)$ is half of the T-cell recruitment rate; and $m$ defines the shape of recruitment function.

Note that as Antia et al [18] point out, "if the parasite persists, the presence of parasite antigens could lead to clonal deletion of parasite-specific immune cells in the thymus." In other words, the clonal deletion, or clonal elimination, is the elimination of T-cells that react with self antigens [42]. Thus, this phenomenon [41,42] explains the negative sign in the second term of Eq. (5).

Moreover, the number of times that immune cells can reproduce is limited [43]. This is a phenomenon observed in epithelial cells by Hayflick and Moorhead [44]. The average number of times that a cell can reproduce is called the "Hayflick limit" [18] and for T-cells, reproduction can be repeated only about 23 times. 
To incorporate the Hayflick limit in the model, a set of $n$ equations represents the reproductive state of the T-cells [18]. The variable $i_{j}$ represents the population of T-cells in the $j$-th stage of the reproduction. Thus, the reproduction cycle of the immune cells is given by:

$$
\begin{aligned}
& \frac{d i_{0}}{d t}=a(\Omega)-\epsilon \frac{\Omega}{\kappa+\Omega} i_{0}-\mu i_{0}, \\
& \frac{d i_{j}}{d t}=2 \epsilon \frac{\Omega}{\kappa+\Omega} i_{j-1}-\epsilon \frac{\Omega}{\kappa+\Omega} i_{j}-\mu i_{j},
\end{aligned}
$$

where, $\epsilon$ is the reproductive stimulus of T-cells; $\kappa$ is a control density to make T-cells to reproduce up to half the maximum value and $\mu$ is the death rate of immune system cells. Thus, in each time step the total population of the immune system cells is

$$
I=\sum_{j=0}^{n} i_{j}
$$

At each time step Eqs. (6) and (7) are concurrently solved and the immune system population is obtained by the sum defined in Eq. (8) for $n=3$.

For the sake of clarity, parameters of the model and their definition can be seen in Table 1. 
Table 1

Parameters of the model.

Par. Definition

Range

References

$q \quad$ mutation rate of $S$ strains

$\left[10^{-8}, 10^{-6}\right]$ generations $^{-1} \quad[45,46]$

$\nu \quad$ reproduction rate of $S$ strain

$[0.36,0.52]$ day $^{-1}$

$\nu_{1} \quad R$ strains reproduction rate $\left(\nu_{1}=w \nu\right)$

$w \in[0.5,1.2]$

$[39,51-53]$

$f \quad$ conversion rate from active to dormant $[0.0,1.0)$ day $^{-1}$

state

$g \quad$ conversion rate from dormant to active $\quad[0.0,1.0)$ day $^{-1}$

state

$\alpha \quad$ antibiotics clearance rate for $S$ strains $\quad[0.003,0.8]$ day $^{-1}$

$[54]$

$\delta \quad$ relative antibiotics efficacy for $R \quad[0.0,1.0]$

pathogens

$\rho \quad$ drug dose reduction factor

$(0.0,1.0)$

$k_{b} \quad$ carrying capacity

400 cell units

$[18]$

$\gamma \quad$ strength of immune system response

0.1

$a_{1} \quad$ recruitment rate of T-cells from thymus

$[0,0.5]$ day $^{-1}$

$a_{2} \quad$ saturation limit for the recruitment of 200 cell units T-cells

$\epsilon \quad$ reproductive stimulus of T-cells

1.0

$\kappa \quad$ reproduction control of T-cells

200 cell units

$\mu \quad$ death rate of immune cells

0.1 day $^{-1}$ 


\section{Results}

To assess the within-host model proposed in the previous section, Eqs. (1-7) are numerically solved. To accomplish this, the fourth order Runge-Kutta method has been used with the initial conditions $S=i_{0}=1$ and $R=S_{d}=R_{d}=0$. Besides, to reduce computational time, we have solved these equations with a Hayflick limit $n=3$, which yields the results as $n=23$. Figures $4(\mathrm{a})$ and 4(b) depict results for $n=3$ and $n=23$, respectively, showing that they are qualitatively equivalent. In all simulations, except when it is mentioned, parameters used are $q=10^{-6}, \nu=0.4$, $\gamma=0.1, g=0.1, k_{b}=400, \omega=0.9, \nu_{1}=\nu \omega, a_{2}=200, m=3.0$, $\kappa=200, \epsilon=1.0$, and $\mu=0.1$.

Since the model is numerically evaluated, two different thresholds have to be defined to solve equations (1-7). The first threshold is the minimum bacterial load ${ }^{\star}, \Omega_{\min }=10^{-3}$, a value used due to limitations in the computational numerical precision. If the host bacterial load, $\Omega$, falls below this threshold $\left(\Omega<\Omega_{\text {min }}\right)$, then $\Omega$ is considered to be zero. In other words, a pathogen load below this value means that the host is clear of TB pathogens, i.e.,

ॠ Note that the bacterial load of each threshold, and for all bacterial populations mentioned in this work, represents a normalized number of pathogens. 
he/she is susceptible, $X$. The second threshold, $\Omega_{\text {lat }}=10^{2}$, sets the amount of pathogens necessary for the transition between TB latency and activity to take place. If a host bacterial load is $\Omega \leq \Omega_{\text {lat }}$, the host is latent, $L_{S}$ or $L_{R}$; otherwise, the host is infectious, $T_{S}$ or $T_{R}$. For instance, this can be seen in Figure 3 from the 0th to approximately 3rd year. In this period, the bacterial load $\left(\Omega<10^{2}\right)$ and the immune system population are higher than their initial values. However, from the 3rd year on, bacterial load increases $\left(\Omega>10^{2}\right)$ whereas the immune system goes to a minimum value remaining steady. This behavior characterizes the transition between the latent and active states.

In our model, cases of co-infection are not considered, in the sense that only one strain defines the type of infection, $S$ or $R$. As an example, one can see the bacterial load as function of time in Figure 4(a). In the first 20 years prior to treatment, $S$ population is higher than $R$ population. Then, even though both strains coexist, we consider the host as being in $T_{S}$ state. Yet, in the 22th year, $R$ population turns to be higher than $S$ population. Again, even both strains are present in the within-host, he/she is considered as $T_{R}$. The same criteria is used to define whether the host is $L_{S}$ or $L_{R}$. 
Figures 1-6 depict numerical solution of our model. For Figs. 13 the system evolves with no health system intervention, $\alpha=0$. Yet, for Figs. 4-6 the system evolves with no antibiotics treatment until the last day of the 19th year. In the first day of the $20 t h$ the treatment is introduced with $\alpha=0.5$ for the cases shown in Figs. 4-6. In the six cases, infection of a susceptible individual, $X$, took place at time $t=0$, with type $S$ bacteria only, thus $S=1$ and $R=0$. The figures show $S, R$ and $I$ populations as function of time. The interpretation of these within-host populations as health states are in the text.

Figures 1-3 depict numerical solution of our model with no health system intervention, $\alpha=0$. In the three cases, infection of a susceptible individual, $X$, took place at time $t=0$, with type $S$ bacteria only, thus $S=1$ and $R=0$. The figures show $S, R$ and $I$ populations as function of time. The interpretation of these within-host populations as health states are in the text.

In Figure 1 one can see a within-host system evolution during 10 years with a recruitment rate of T-cells from thymus $a_{1}=$ 0.41. This value allows the host immune response to be strong enough to clear the infection. Therefore, one month after infection has occurred, the immune system starts to control the pathogen 


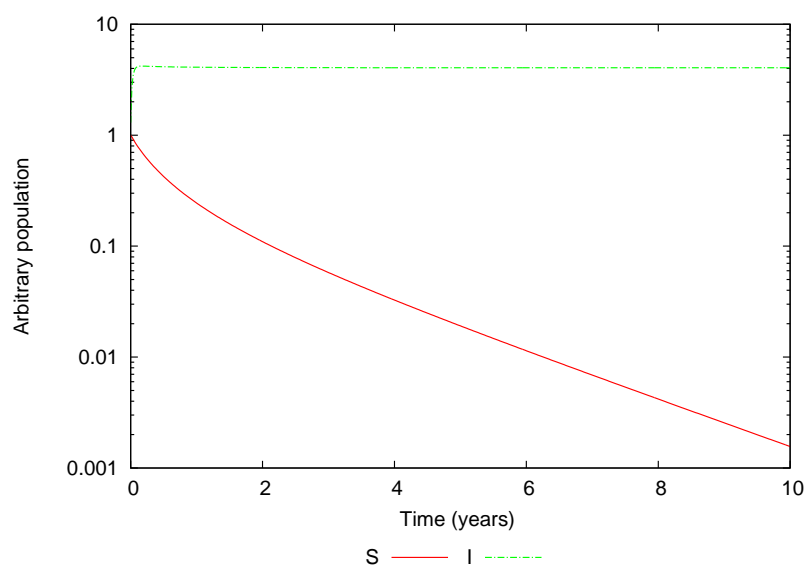

Fig. 1. Arbitrary population $S$ (solid line) and $I$ (dotted dashed line) as function of time for a host infected at $t=0$. Host is clear of TB pathogens 10 years after the infection. Vertical axis is on a logarithmic scale. Parameters: $a_{1}=0.41, f=0.2$, $\alpha=0$.

reproduction. Close to the end of the tenth year, the host system is completely clear of the infection. Note that no antibiotics was used in this case, $\alpha=0$.

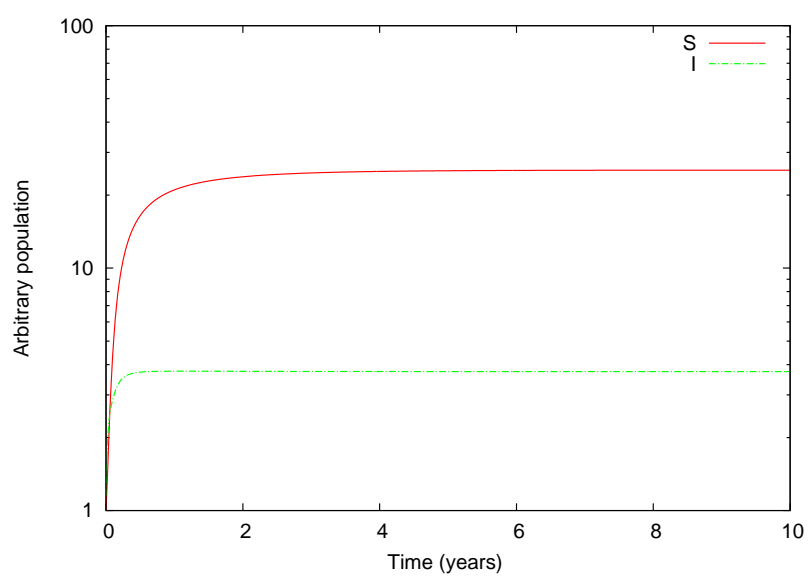

Fig. 2. Arbitrary population $S$ (solid line) and $I$ (dotted dashed line) as function of time for a host infected at $t=0$. Host is in an latent state of TB, $L_{S}$. Horizontal dotted line represents the latency threshold, $\Omega_{l a t}=10^{2}$. Vertical axis is on a logarithmic scale. Parameters: $a_{1}=0.27, f=0.2, \alpha=0$. 
Figure 2 depicts the evolution of the within-host system for $a_{1}=$ 0.27. From the first month until the second one after the infection has happened, the $S$ population grows. This bacterial growth is followed by the immune response, which increases the $I$ population as well. Then, by approximately in the fourth month the infection is under control due to the immune system response. Once the host immune system has controlled the infection, but not cleared it, and $\Omega<\Omega_{\min }$, the latent stage starts.

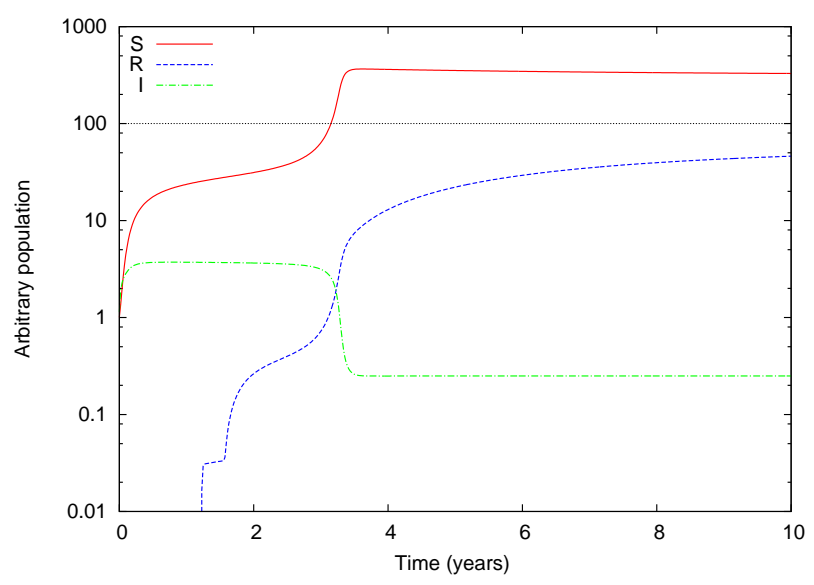

Fig. 3. Arbitrary population $S$ (solid line), $R$ (dashed line) and $I$ (dotted dashed line) as function of time for a host infected at $t=0$. Host is in an active state of $\mathrm{TB}, T_{S}$. Horizontal dotted line represents the latency threshold, $\Omega_{l a t}=10^{2}$. Vertical axis is on a logarithmic scale. Parameters: $a_{1}=0.251, f=0.2, \alpha=0$.

The evolution of a TB infection from a susceptible state to an active state can be seen in Figure 3. In this case, $a_{1}=0.251$, which represents a lower migration rate of T-cells in comparison to the previous cases, figures 1 and 2. The reduction in this parameter 
value completely changes the outcome of the system. Approximately one year after infection starts, the immune system is no longer capable of controlling the bacterial reproduction. In the beginning of the second year, $R$ type pathogens start to increase to significant values. Thus, bacterial load, $\Omega$, reaches values that suppress the immune system, $I$, to minimum values.

Then, by around the 3rd year, $S$ type population crosses the latency limit (dashed line), $\Omega_{\text {lat }}$. From this moment, the host progress from the latent state, $L_{S}$, to the active state, $T_{S}$. Note that no antibiotics are being used, therefore, the rise of resistant strains is due only to the mutation of sensitive strains. At the 10th year, $\approx 12 \%$ out of the total pathogen population is composed of $R$ type bacteria.

The impact of antibiotic treatment on the emergence of drug resistance can be seen in Figure 4. An individual with active tuberculosis with type $S$ pathogen, $T_{S}$, starts the treatment in the first day of the 20th year. The drug is taken on a daily basis during 180 days with clearance rate $\alpha=0.5$.

An abrupt fall in the $S$ population occurs immediately after the treatment begins. About one month after the use of drugs starts, 


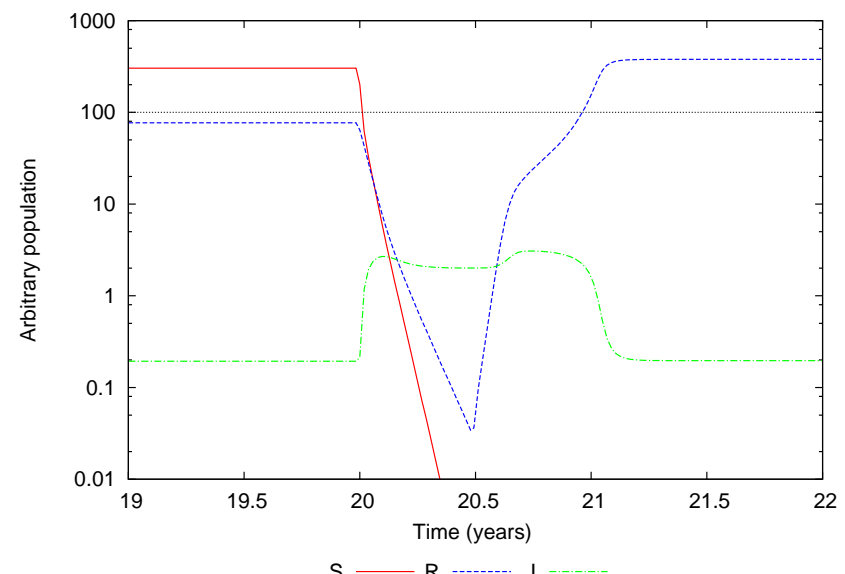

(a) $n=3$

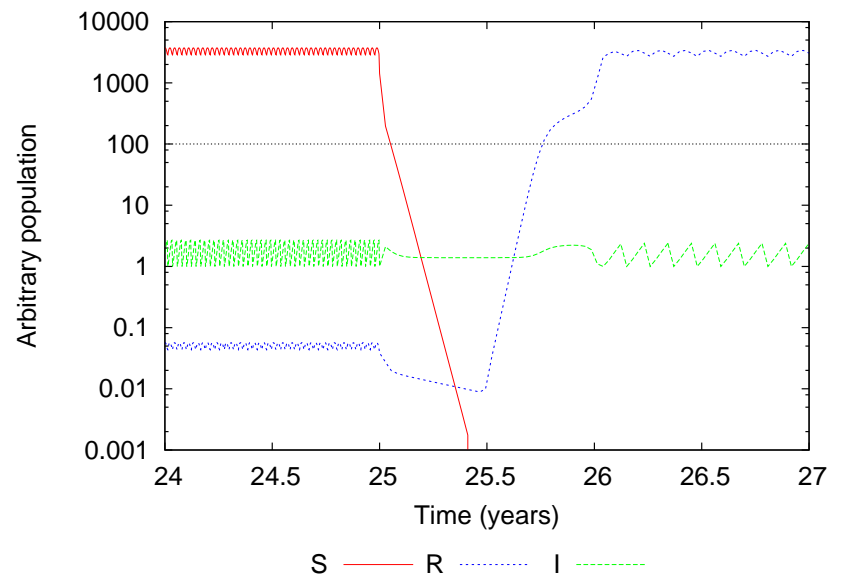

(b) $\mathrm{n}=23$

Fig. 4. Arbitrary population $S$ (solid line), $R$ (dashed line) and $I$ (dotted dashed line) as function of time. Treatment starts at the first day of the $t=20$ th year for (a) $n=3$ and $t=25$ for (b) $n=23$. Antibiotic doses are applied on a daily basis during 180 days. Host is initially in $T_{S}$ state but after the use of drugs he/she becomes $T_{R}$. Horizontal dashed line represents the latency threshold, $\Omega_{\text {lat }}=10^{2}$. Vertical axis is on a logarithmic scale. In addition, we present the results for $n=23$ and they are qualitatively the same as $n=3$, since $S$ population vanishes and $R$ is the highest population. Parameters: (a) $n=3, a_{1}=0.20, f=0.1, \alpha=0.5, \delta=0.5$ (b) $n=23, k_{b}=10000, \alpha=0.8, \delta=0.2$. 
sensitive pathogens are completely cleared from the system. Because of the relative efficacy of antibiotics, $\delta=0.5$, the $R$ population also decreases rapidly. Nevertheless, resistant strains are not completely cleared from this system. These two phenomena are followed by the increase in the immune system population, $I$.

Also in Fig. 4, as soon the treatment ends, the immune system response is not enough to inhibit the $R$ strain's growth. As long as the remaining resistant pathogens do not have to compete with sensitive strains $(S=0)$, their growth is faster. Thus, around the first month of the 21th year, this host becomes $T_{R}$. This is a typical case of emergence of drug resistance due to the use of antibiotics.

Figure 5 depicts a within-host system with the same initial conditions as in fig. 4. The only difference in this case is a slightly higher recruitment rate of T-cells from thymus, $a_{1}=0.24$. Again, $S$ strains vanish due to the drugs, which gives a competitive advantage to $R$ strains. A rapid growth of resistant pathogens initiates as soon as treatment ends. Yet, a higher $a_{1}$ allows a stronger immune system response to fight against resistant strains. The final result is an equilibrium between $R$ and $I$ populations. Once the $R$ population is lower than the latency limit, this individual 


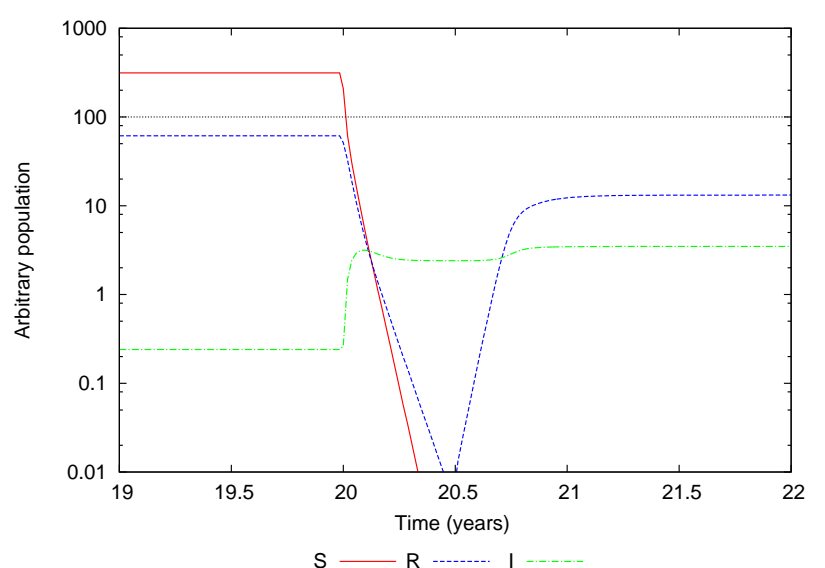

Fig. 5. Arbitrary population $S$ (solid line), $R$ (dashed line) and $I$ (dotted dashed line) as function of time. Treatment starts at the first day of the $t=20$ th year. Antibiotic doses are applied on a daily basis during 180 days. Host is initially in $T_{S}$ state but after the use of drugs he/she becomes $L_{R}$. Horizontal dashed line represents the latency threshold, $\Omega_{l a t}=10^{2}$. Vertical axis is on a logarithmic scale. Parameters: $a_{1}=0.24, f=0.1, \alpha=0.5, \delta=0.5$.

becomes latent, $L_{R}$.

Results shown in Figure 6 are for a system under the same initial conditions of figures 4 and 5 . The conversion rate to the dormant stage is reduced to $f=0.05, a_{1}=0.23$. The change in these parameters combined with the effect of antibiotics are enough to eliminate $S$ and $R$ type pathogens completely. This is a case where the host is cured due to the use of antibiotics.

The numerical solutions of equations (1-7), shown in Figures 1-6, do not reveal the important role of entry $(f)$ and exit $(g)$ of the dormancy state. We note that dormant bacteria do not reproduce 


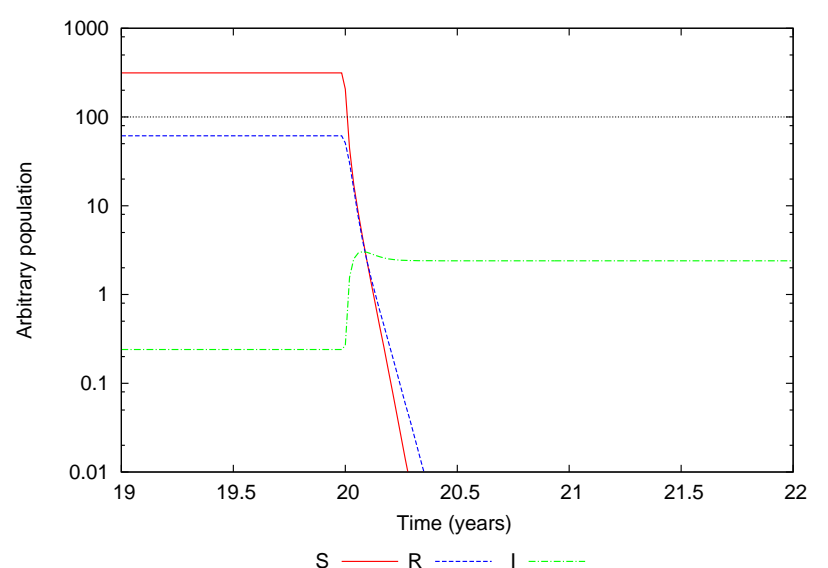

Fig. 6. Arbitrary population $S$ (solid line), $R$ (dashed line) and $I$ (dotted dashed line) as function of time. Treatment starts at the first day of the $t=20$ th year. Antibiotic doses are applied on a daily basis during 180 days. Host is initially in $T_{S}$ state but after the use of drugs he/she becomes $L_{R}$. Horizontal dashed line represents the latency threshold, $\Omega_{l a t}=10^{2}$. Vertical axis is on a logarithmic scale. Parameters: $a_{1}=0.23, f=0.05, \alpha=0.5, \delta=0.5$.

and thus they also are not affected by antibiotics. Thus, in the next subsection, the impact of this state in the outcome of the within-host system is tested by using a set of antibiotic protocols.

\subsection{Protocols}

In this section, the use of antibiotics is analyzed by testing different types of protocols. All the results are obtained for the withinhost model applied to only one host. Outcomes of each protocol are shown in diagrams for parameters $f$, conversion rate from active to dormant state, versus $a_{1}$, and recruitment rate of T-cells 
from the thymus. Three types of protocols are implemented: standard (SP), intermittent (IP) and oscillating intermittent (OIP). For the three types of protocols, Eqs. (1-8) are solved numerically for several combinations of parameters $f$ and $a_{1}$. Initially, the system evolves with no intervention during a period equivalent to 75 years. After this period, steady state is reached, the treatment is applied and the system starts to evolve again. Outcomes shown in all diagrams are obtained 25 years after the beginning of the treatment.

However, to check the evolution of the system with no medical intervention, we initially present a diagram without treatment. Figure 7 depicts a phase diagram for individuals without treatment. In this case, three outcomes are possible: $i$ ) to be naturally cured; $i i)$ to become latent with only type $S$ pathogen $\left(L_{S}\right)$ and, iii) to become ill with TB with type $S$ bacteria only, $\left(T_{S}\right)$. One can see clearly in the diagram that the existence of a dormant stage (variation of $f$ ), without the use of antibiotics, does not affect the outcomes. The different results are due only to the recruitment rate of immune cells coming from the thymus, $a_{1}$. Note that for latent and active states, only sensitive pathogens are present. This is the reason that if no antibiotics is used, the 
possibility for the $R$ type to arise is due only to mutation of $S$ during strain reproduction.

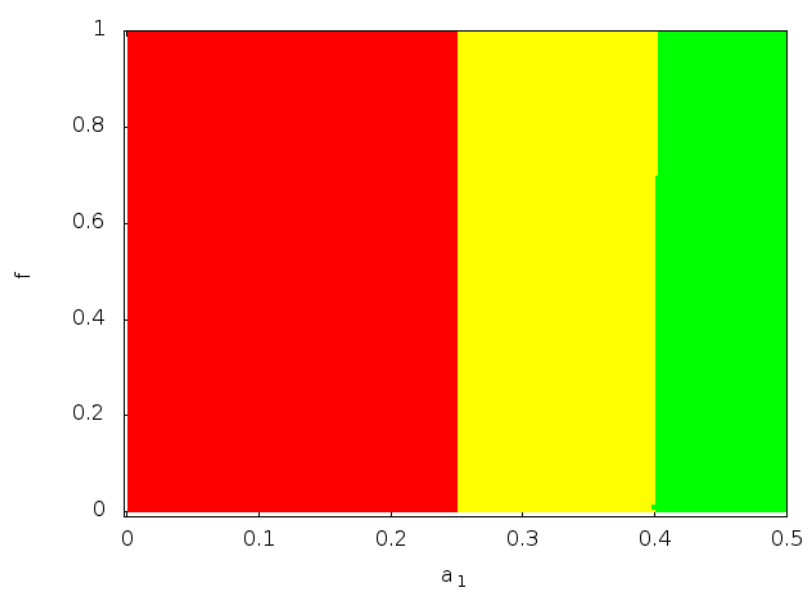

Fig. 7. Phase diagram of the conversion rate from active to dormant state, $f$, versus the recruitment rate of T-cells from thymus, $a_{1}$, without treatment $(\alpha=0)$. Each color represents a state: green, $X$; yellow, $L_{S}$; red, $T_{S}$.

\subsubsection{Standard Protocol (SP)}

The standard protocol (SP) is characterized by the fact that individuals are getting antibiotics on a daily basis during 180 days ( 6 months). Figure 8a depicts the phase diagram for the standard protocol with $\alpha$ and $\delta$ kept constant during the treatment period. In this plot, $\delta=0.8$, what means that the actual clearance rate of $R$ strains is not $\alpha$, but $\delta \alpha$. The use of a relative efficacy, $\delta \neq 0$, can be interpreted as a multi-drug antibiotic treatment. Due to this combination of parameters, only four states of TB are present in the diagram. If both $S$ and $R$ are above threshold, 
we considered as an $S$ strain for the effect of representation in the diagram, hereafter.

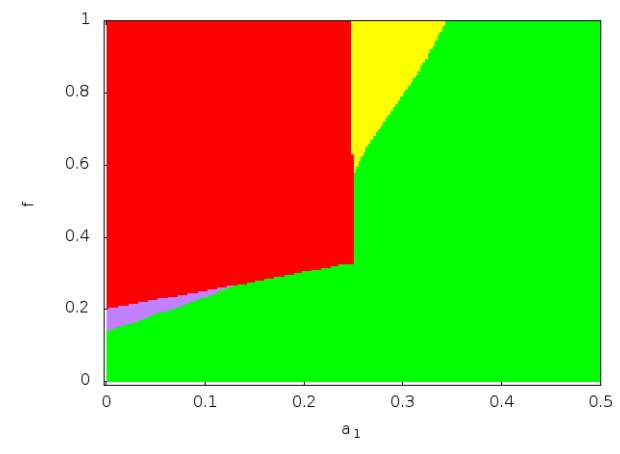

(a) $\alpha=0.8, \delta=0.8$

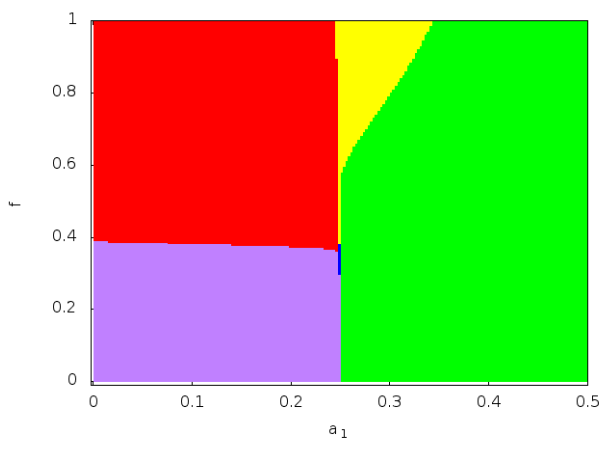

(b) $\alpha=0.8, \delta=0.0$

Fig. 8. Phase diagram of the conversion rate from active to dormant state, $f$, versus the recruitment rate of T-cells from thymus, $a_{1}$, using standard protocol (SP). Note how dormancy changes the outcomes. Each color represents a state: green, $X$; yellow, $L_{S}$; blue, $L_{R}$; red, $T_{S}$; purple, $T_{R}$. In this diagram and in the following ones, if $S$ and $R$ are above threshold, we considered as an $S$ state for representation.

As expected, on the other hand, there is a strong reduction in $T_{S}$ cases (red area of the diagram) due to the effect of drugs in sensitive strains. On the other hand, the emergence of drug resistance problem, i.e, $T_{R}$ cases (purple area) is now present. Resistant strains do not arise for $f \lesssim 0.15$ no matter the value of $a_{1}$. For values of $f \gtrsim 0.20$, the influence of the dormancy in the emergence of resistant strains becomes evident. As $a_{1}$ increases, more immune cells from thymus are available in the host system. Thus, to allow the existence of $R$ type pathogen, the conversion rate to the dormant state, $f$, also has to be increased. Cells in 
a dormant state are not affected by immune response nor by antibiotics.

The plot of Fig. 8b shows the results of a SP protocol treatment with one drug. Since resistant strains are not affected only by the treatment $(\delta=0.0)$, the emergence of drug resistance is high (purple area). Cases of latency with resistant pathogens, $L_{R}$, even small, (blue area) can be seen in the diagram. The prevalence of $T_{S}$ cases occurs only for $f \gtrsim 0.40$ and for $a_{1} \lesssim 0.25$. If $a_{1} \gtrsim$ 0.25 , the immune system is strong enough to eliminate all type of pathogens (no active cases) or to allow the latency state (yellow area).

\subsubsection{Intermittent Protocol (IP)}

The intermittent protocol (IP) is characterized by the application of drugs in an intermittent fashion. More specifically, individuals get antibiotics during $y$ days. These doses are interrupted for another $n$ days. This cycle is repeated during a predetermined period, $p$. The relation $y / n / p$ defines the type of intermittent protocols that is being implemented.

Figure 9 a depicts a phase diagram for an IP:3/6/540. The treatment is applied during 3 days, it is interrupted during 6 days 
and this cycle is repeated by 540 days. Note that a 3/6/540 cycle guarantees that the total amount of doses is the same of a standard protocol.

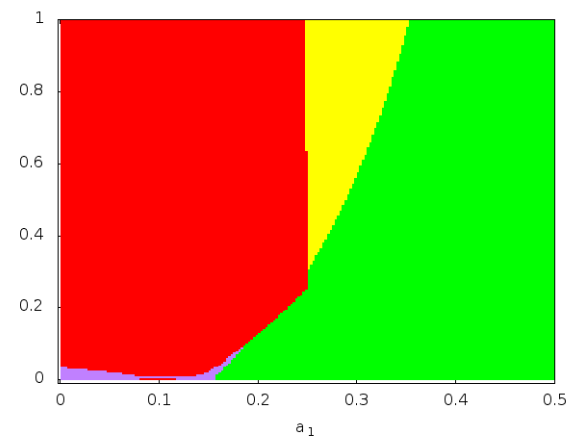

(a) IP:3/6/540, $\alpha=0.8, \delta=0.8$

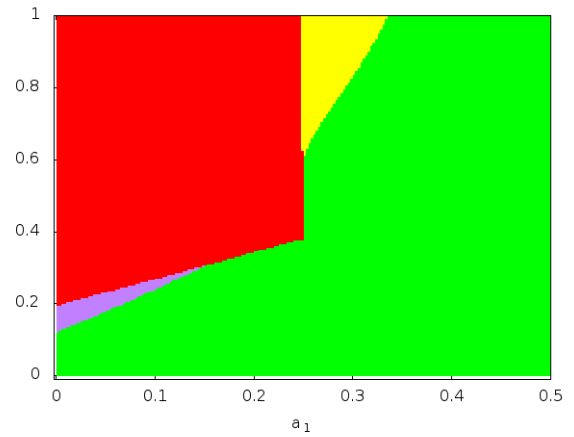

(b) IP: $6 / 1 / 210, \alpha=0.8, \delta=0.8$

Fig. 9. Phase diagram of the conversion rate from active to dormant state, $f$, versus the recruitment rate of T-cells from thymus, $a_{1}$, with intermittent protocol IP:3/6/540. The cycle is 3 days with treatment and 6 days without. Note that dormancy changes the outcomes. Each color represents a state: green, $X$; yellow, $L_{S}$; blue, $L_{R}$; red, $T_{S}$; purple, $T_{R}$.

Fig. 9a shows that the IP:3/6/540 presents a worse outcome compared to the SP protocols, show in Figs. 8a and 8b. Red area of the diagram, representing $T_{S}$, is bigger than in the case of SP protocols. Besides, IP:3/6/540 presents emergence of drug resistance and it also allows the existence of a larger latency region, $L_{S}($ yellow area)

Results for an IP:6/1/210 are displayed in Figure 9b. Again, for the sake of comparison, the whole cycle is 210 days, to keep the 
same amount of doses of a SP. The IP:6/1/210 presents better results than the IP:3/6/540 (Fig. 9a), a similar outcome with $\delta=0.8$ (Fig. 9b) and better than SP with $\delta=0.0$ (Fig. 8b). This protocol has a smaller area for $T_{S}$ cases in comparison to the IP:3/6/540. Nevertheless, in the diagram of Fig. $9 \mathrm{~b}, T_{R}$ region is larger than in the Fig. 9a.

\subsubsection{Oscillating Intermittent Protocol (OIP)}

The oscillating intermittent protocol (OIP) is similar to intermittent protocol discussed previously. As mentioned before, $\alpha$ is the rate at which antibiotics kill $S$ type bacteria. The main feature of OIP is that the value of $\alpha$ oscillates during the treatment. Thus, this parameter becomes time dependent and obeys the relation $\alpha_{t}=\rho \alpha_{t-1}$, where $\rho$ is the drug dose reduction factor. For instance, OIP:3/6/540 means that the drug is: taken during three days ( $\alpha$ is constant)/suspended by six days ( $\alpha$ decays)/the whole treatment lasts 540 days.

Using OIP, we aim to simulate two different types of conditions. Firstly, a scenario where the drug is not completely cleared from the host system in a period of one day. Thus we can observe how different amounts of the antibiotics, inside the host, can affect the 
disease. Secondly, a kind of antibiotic which is taken up by the host during a period with different concentration. By concentration, we mean that each dose could be made of pills of different sizes.

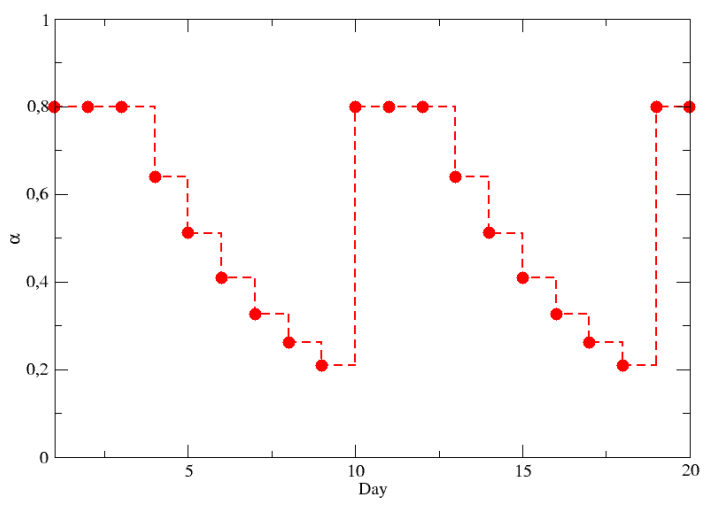

Fig. 10. Oscillation of $\alpha_{t}$ as function of time for intermittent protocols. At the first day of treatment, $t=0, \alpha_{t=0}=0.8$ and it is reduced daily by a factor $\rho=0.8$. Note that after a period of 9 days the parameter $\alpha_{t}$ returns to its initial value $\alpha_{t=0}$.

In Figure 10, the behavior of $\alpha_{t}$ as function of time in days, with a reduction factor, $\rho=0.8$, is shown. In other words, $\alpha_{t}$ is reduced $80 \%$ in relation to the value of the previous day. This daily reduction occurs in a period without treatment and then it returns to its initial value again.

Figure 11a depicts results for an OIP:3/6/540, i.e, 3 days getting antibiotics, 6 days without antibiotics, during a 540 days period. Again, the amount of doses taken is the same as in the standard 
protocol.

The $\alpha_{t}$ oscillation is implemented similarly to method shown in figure 10. In the first day, $\alpha_{t=0}=0.8$ and then, in the second day, it is reduced for a value of $\alpha_{t=1}=\rho \alpha_{t=0}=0.64$, with $\rho=0.8$. In the third day, the antibiotics is again reduced for the value $\alpha_{t=2}=\rho \alpha_{t=1}=0.512$. Finally, the treatment is interrupted $(\alpha=$ 0) during 6 days and this whole cycle is repeated for 540 days.

The largest area in diagram of Fig. 11a is related to susceptible cases, $X$. In comparison to the the SP and IP protocols tested in the previous subsections, OIP:3/6/540 has the best outcome. $T_{S}$ and $T_{R}$ cases emerge only for small values of $a_{1}$ and high values of $f$. Note that even though the number of doses is the same as SP protocol, the different values of concentrations implies that the total quantity of antibiotics is smaller. In other words, an OIP protocol presents better results and besides it is less expensive.

Figures 11b depicts an OIP:3/6/540 with a reduction factor $\rho=$ 0.5. This protocol is similar to the fig. 11a except by the reduction factor which is now 0.5. This means that the concentration of antibiotics in the host system is reduced by $50 \%$ in relation to the previous day. A simple $50 \%$ reduction in the concentration of 


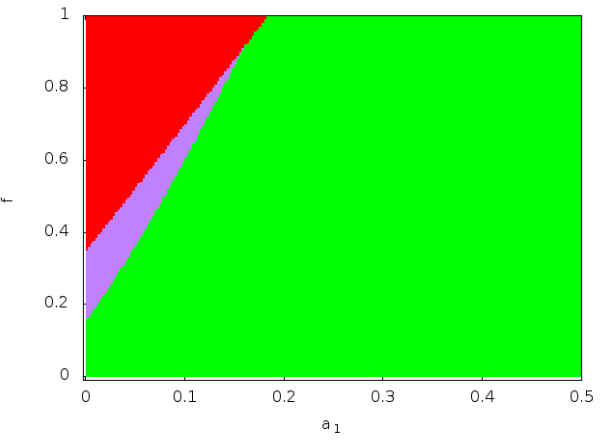

(a) OIP:3/6/540, $\delta=0.8, \rho=0.8$

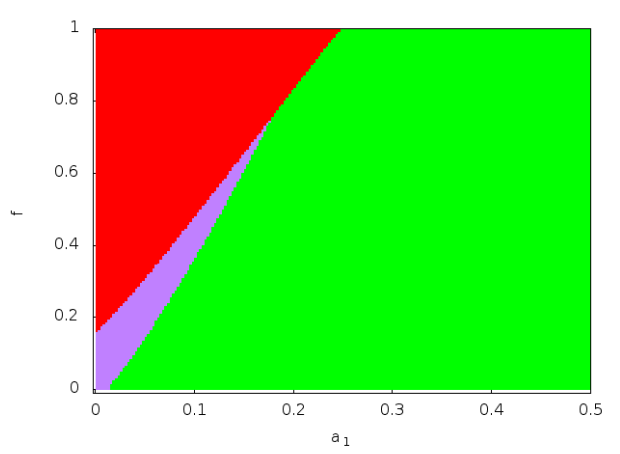

(c) OIP: $1 / 6 / 540, \delta=0.8, \rho=0.8$

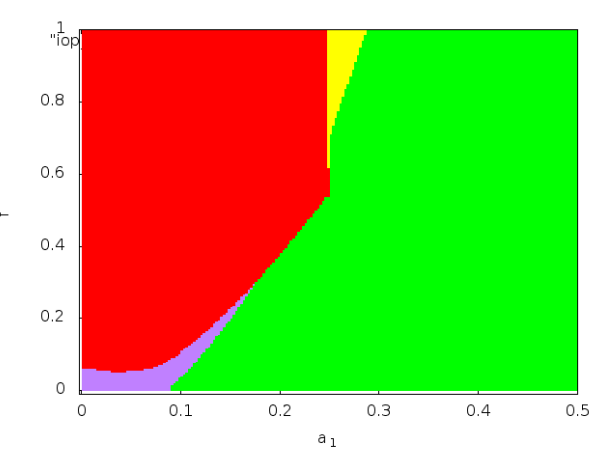

(b) OIP:3/6/540, $\delta=0.8, \rho=0.5$

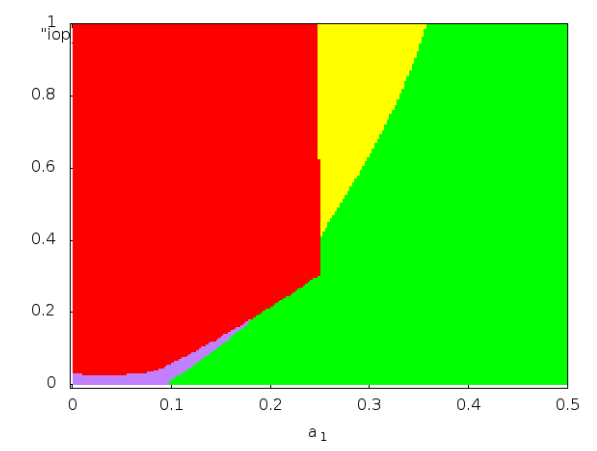

(d) OIP:3/6/360, $\delta=0.8, \rho=0.5$

Fig. 11. Phase diagram of the conversion rate from active to dormant state, $f$, versus the recruitment rate of T-cells from thymus, $a_{1}$ for oscillating intermittent protocols with $\alpha_{t=0}=0.8$. Note how dormancy changes the outcomes. Each color represents a state: green, $X$; yellow, $L_{S}$; blue, $L_{R}$; red, $T_{S}$; purple, $T_{R}$.

antibiotics provokes worse results, as seen in fig. $11 \mathrm{~b}$.

In an OIP:3/6/540 with $\rho=0.5$, though $T_{R}$ cases area has just been displaced in the diagram, $T_{S}$ cases area is clearly larger than in OIP:3/6/540 with $\rho=0.8$. The difference in the concentration of antibiotics does not affect the emergence of drug resistance. On the other hand, this reduced concentration allows the persistence of $S$ strains in a larger region of the diagram. 
Figure 11c depicts results for an OIP:1/6/540 with $\rho=0.8$. The cycle $1 / 6$ for the whole 540 days period means that only $1 / 3$ of SP doses will be used. For this protocol, each individual will only take one dose of antibiotics once in a week. For this protocol, results are between those obtained in figs. 11a and 11b. Emergence of drug resistance is higher, despite the one week dose.

In Fig. 11d is plotted an OIP:3/6/360 with a reduction factor $\rho=0.8$. The reduction of the period of treatment from 540 days to 360 days means that $2 / 3$ of the total doses are being used. In this diagram regions related to $T_{S}$ is bigger whereas $T_{R}$ region is similar to the protocol form fig. 11c. Sentitive TB persists and the emergence of drug resistance still takes place. Even though the OIP:3/6/360 has worse outcomes compared to OIP:3/6/540 (fig. 11a), its results are better than the SP.

In order to understand why the OIP is so efficient, we present in Fig. 12, how the population of $\mathrm{S}$ is changed for both protocols: IP and OIP. It is clear that the population decreases in a faster rate for the OIP than IP. 


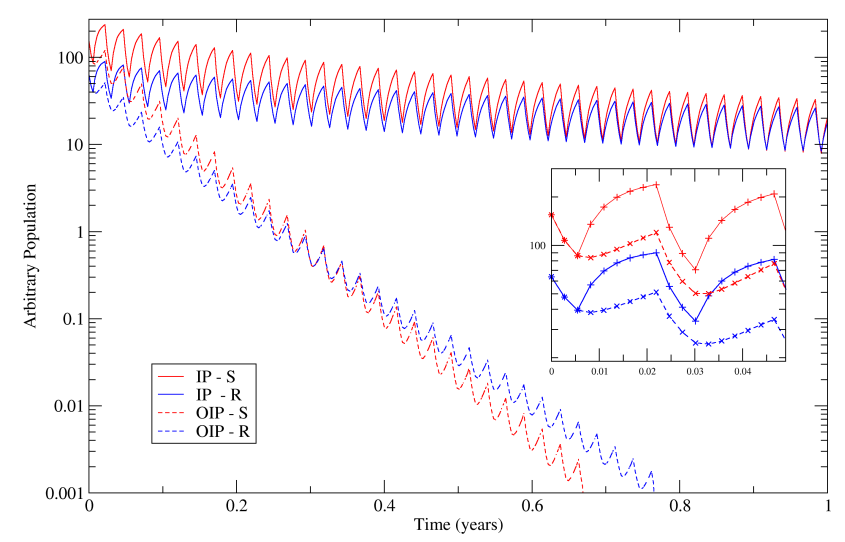

Fig. 12. Arbitrary populations of $S$ and $R$ strains for two different protocols IP:3/6/540 and OIP:3/6/540. Inset: Zoom for the period between 0.00 and 0.04 years. Although one can see a decreasing trend in both protocols, it is more pronounced in the OIP. Parameters are: $\alpha=\delta=0.8$ and $f=a_{1}=0.2$.

\section{Discussion}

In this paper we propose a within-host TB model with the interplay among sensitive/resistant pathogens, immune system cells, bacterial dormancy and antibiotics. In contrast to existing withinhost models, here resistant strains and bacterial dormancy are combined simultaneously in our model. The host's health state is defined according to his/her pathogen load, allowing more precise quantification of the distance between TB latency and activity. This is important to determine the necessity of an antibiotic treatment as well as its urgency.

A set of coupled ordinary differential equations describes the 
within-host model dynamics. Numerical solutions varying the amount of antibiotic doses and their periodicity define three different protocols: standard (SP), intermittent (IP) and oscillating intermittent (OIP) protocols. They are analysed for a range of values for the rate of T-cells migration from thymus and pathogen dormancy rates.

Although there are the remnants of active cases with drug resistant strains, $T_{R}$, the oscillating intermittent protocol (OIP) leads to a greater number of susceptible individuals, $X$. This is robust under the variation of the drug dose reduction factor, $\rho=0.8$ and $\rho=0.5$, with relative efficacy $\delta=0.8$ for both cases. It is the most effective protocol analysed. The effectiveness of OIP is followed by SP, also with $\delta=0.8$. Even though the latter protocol presents a lower amount of susceptibles, $X$, it only presents latent drug resistant cases, $L_{R}$. Finally, the least effective protocol is IP, with $\delta=0.8$, since active cases, $T_{R}$, are present instead of resistant latent ones, $L_{R}$. We speculate that OIP may also be the most effective protocol for other diseases, implying in a lower treatment cost. 


\title{
5 Acknowledgments
}

\author{
A.L.E. and C.T.B. wish to thank Natural Sciences and Engineer- \\ ing Research Council of Canada (NSERC) and Canadian Insti- \\ tutes of Health Research (CIHR) for financial support. A.S.M. ac- \\ knowledges the Brazilian agency CNPq (305738/2010-0) for sup- \\ port. B.C.T.C. acknowledges the Brazilian agency CNPq (127151/2012- \\ 5) for support. This work is partially supported by Brazilian agen- \\ cies CNPq, FAPERJ and CAPES.
}

\section{References}

[1] C. D. Deangelis and A. Flanigin. Tuberculosis - a global problem requiring a global solution. JAMA, 293:2793-2794, 2005.

[2] World Health Organization. Tuberculosis fact sheet nr. 104. http://www . who. int/mediacentre/factsheets/fs104/en/index.html, Nov 2010.

[3] B. R. Bloom and C. J. L. Murray. Tuberculosis: commentary on a reemergent killer. Science, 257:1055-1064, 1992.

[4] M. A. Behr et al. Transmission of mycobacterium tuberculosis from patients smear-negative for acid-fast bacilli. Lancet., 353(9151):444-449, 1999.

[5] W. W. Nazaroff M. Nicas and A. Hubbard. Toward understanding the risk of secondary airborne infection: emission of respirable pathogens. J. Occup. Environ. Hyg., 2(3):143-154, 2005. 
[6] Thomas House and Matt J. Keeling. The impact of contact tracing in clustered populations. PLoS Comput Biol, 6(3):e1000721, 032010.

[7] Mercedes Pascual. Computational ecology: From the complex to the simple and back. PLoS Comput Biol, 1(2):e18, 072005.

[8] S. M. Blower et al. The intrinsic transmission dynamics of tuberculosis epidemics. Nature Medicine, 8(1):815-821, 1995.

[9] P. M. Small S. M.Blower and P. Hopewell. Control strategies for tuberculosis epidemics: new models for old problems. Science, 273:497-500, 1996.

[10] T. C. Porco T. S. M. Blower and T. Lietman. Tuberculosis: the evolution of antibiotic resistance and the design of epidemic control strategies. Mathematical Models in Medical and Health Sciences. Eds Horn, Simonett, Webb. Vanderbilt University Press, 1998.

[11] S. M. Blower and C. L. Daley. Problems and solutions for the stop tb partnership. Lancet. Infect. Dis., 2:374-376, 2002.

[12] K. Koelle S. M. Blower and T. Lietman. Antibiotic resistance - to treat...(or not to treat)? Nature Medicine, 5(4):358-359, 1999.

[13] T. C. Porco and S. M. Blower. Quantifying the intrinsic transmission dynamics of tuberculosis. Theor. Popul. Biol., 54:117-132, 1998.

[14] S. M. Blower et al. The intrinsic transmission dynamics of tuberculosis epidemics. Nature Medicine, 1(8):815-821, 1995.

[15] Justino Alavez-Ramirez et al. Within-host population dynamics of antibioticresistant M. Tuberculosis. Mathematical Medicine and Biology, 24:35-56, 2006.

[16] C. Castillo-Chavez and Z. Feng. To treat or not to treat: the case of tuberculosis. J. Math. Biol., 35:629-659, 1997. 
[17] C. Castillo-Chavez and Z. Feng. Mathematical Models for the Disease Dynamics of Tuberculosis. In: Axelrod, O.D., Kimmel, M. (eds.) Advances In Mathematical Population Dynamics - Molecules, Cells and Man, pp. 629-656. World Scientific Press, Singapore, 1998.

[18] Jacob C. Koella Rustom Antia and Veronique Perrot. Models of the withinhost dynamics of persistent mycobacterial infections. Proc. R. Soc. Lond. B, 263:257-263, 1996.

[19] S. Marino, D. Sud, H. Plessner, P.L. Lin, J. Chan, et al. Differences in reactivation of tuberculosis induced from anti-tnf treatments are based on bioavailability in granulomatous tissue. PLoS Comput Biol, 3(10):e194, 2007.

[20] Sud D., Bigbee C., Flynn J.L., and Kirschner D.E. Contribution of cd8+ t cells to control of mycobacterium tuberculosis infection. J Immunol., 176(7):4296$314,2006$.

[21] M. Fallahi-Sichani, M. El-Kebir, S. Marino, D.E. Kirschner, and J.J. Linderman. Multiscale computational modeling reveals a critical role for tnf-alpha receptor 1 dynamics in tuberculosis granuloma formation. The Journal of Immunology, 186(6):3472, 2011.

[22] M.A. Nowak and R.M.C. May. Virus Dynamics: Mathematical Principles of Immunology and Virology. Oxford University Press, 2000.

[23] Rustom Antia, Bruce R. Levin, and Robert M. May. Within-Host Population Dynamics and the Evolution and Maintenance of Microparasite Virulence. The American Naturalist, 144(3), 1994.

[24] Lauren Ancel Meyers, Bruce R. Levin, Anthony R. Richardson, and Igor Stojiljkovic. Epidemiology, hypermutation, within-host evolution and the virulence of neisseria meningitidis. Proceedings of the Royal Society of London. Series B: Biological Sciences, 270(1525):1667-1677, 2003. 
[25] Kasia A. Pawelek, Giao T. Huynh, Michelle Quinlivan, Ann Cullinane, Libin Rong, and Alan S. Perelson. Modeling within-host dynamics of influenza virus infection including immune responses. PLoS Comput Biol, 8(6):e1002588, 06 2012 .

[26] Fabio Luciani and Samuel Alizon. The evolutionary dynamics of a rapidly mutating virus within and between hosts: The case of hepatitis c virus. PLoS Comput Biol, 5(11):e1000565, 112009.

[27] S. Alizon and van M. Baalen. Acute or chronic? within-host models with immune dynamics, infection outcome, and parasite evolution. Am Nat., 172(6):E244-56, December 2008.

[28] Gesham Magombedze Edward T. Chyiaka and Lawrence Mutimbu. Modelling within host parasite dynamics of schistosomiasis. Computational and Mathematical Methods in Medicine, 11(3):255-280, Semptember 2010.

[29] Carl T. Bergstrom Vitaly V. Ganusov and Rustom Antia. Within-host population dynamics and the evolution of microparasites in a heterogeneous host population. Evolution, 56(2):213-223, 2002.

[30] A. J. Grant, O. Restif, T. J. McKinley, M. Sheppard, D. J. Maskell, and P. Mastroeni. Modelling within-host spatiotemporal dynamics of invasive bacterial disease. PLoS Biol., 6(4):e74, April 2008.

[31] Janis E. Wigginton and Denise Kirschner. A model to predict cell-mediated immune regulatory mechanisms during human infection with mycobacterium tuberculosis. The Journal of Immunology, 166(3):1951-1967, 2001.

[32] Sarah L. Kinnings, Nina Liu, Nancy Buchmeier, Peter J. Tonge, Lei Xie, and Philip E. Bourne. Drug discovery using chemical systems biology: Repositioning the safe medicine comtan to treat multi-drug and extensively drug resistant tuberculosis. PLoS Comput Biol, 5(7):e1000423, 072009. 
[33] Bálint Mészáros, Judit Tóth, Beáta G. Vértessy, Zsuzsanna Dosztányi, and István Simon. Proteins with complex architecture as potential targets for drug design: A case study of mycobacterium tuberculosis. PLoS Comput Biol, 7(7):e1002118, 072011.

[34] Samiul Hasan, Sabine Daugelat, P. S. Srinivasa Rao, and Mark Schreiber. Prioritizing genomic drug targets in pathogens: Application to mycobacterium tuberculosis. PLoS Comput Biol, 2(6):e61, 062006.

[35] L. Ramakrishnan P.L. C. Small and S. Falkow. Remodeling schemes of intracellular pathogens. Science, Wash., 263(637-638), 1994.

[36] J. M. Grange. The mystery of mycobacterial persistor. Tubercle Lung Dis., 73:645-675, 1992.

[37] P. W. Roche W. J. Britton and N. Winter. Mechanisms of persistence of mycobacteria. TIM, 2:284-288, 1994.

[38] Bhatter P. amd Chatterjee A., D'souza D., and Mistry N. Tolani M. Estimating fitness by competition assays between drug susceptible and resistant mycobacterium tuberculosis of predominant lineages in mumbai, india. PLoS ONE, 7(3):e33507, 2012.

[39] B. Sommers T. Cohen and M. Murray. The effect of drug resistance on the fitness of Mycobacterium tuberculosis. Lancet Infect. Dis., 3:13-21, 2003.

[40] B. Björkholm et al. Mutation frequency and biological cost of antibiotic resistance in Heliobacter pylori. Proc. Natl. Acad. Sci. USA, 98:14607-14612, 2001.

[41] P. Parham. The Immune System. The Immune System. Garland Science, 2005.

[42] Alan S. Perelson and Gerard Weisbuch. Immunology for physicists. Reviews of Modern Physics, 69:1219-1267, 1997. 
[43] M. A. Newman N. L. Perillo, R. L. Walford and R. B. Effros. Human T lymphocytes possess a limited in vitro life span. Exp. Gerontol., 24:177-187, 1988.

[44] L. Hayflick and P. S. Moorhead. The serial cultivation of human diploid cell strains. Exp. Cell Res., 25:585-621, 1961.

[45] T. Shimao. Drug resistance in tuberculosis control. Tubercle, 68:5-15, 1987.

[46] J. Werngren and S. E. Hoffner. Drug-susceptible mycobacterium tuberculosis beijing genotype does not develop mutation-conferred resistance to rifampin at an elevated rate. J. Clin. Microbiol., 41:1520-1524, 2003.

[47] Q. Li, C. C. Whalen, J. M. Albert, R. Larkin, L. Zukowski, M. D. Cave, and R. F. Silver. Differences in rate and variability of intracellular growth of a panel of mycobacterium tuberculosis clinical isolates within monocyte model. Infect. Immun., 70:6489-6493, 2002.

[48] M. J. Zhang, J. Gong, Z. Yang, B. Samtem, M. D. Cave, and P. F. Barnes. Enhanced capacity of a widespread strain of mycobacterium tuberculosis to grow in human monocytes. J. Infect. Dis., 179:1213-1217, 1999.

[49] R. J. North, L. Ryan, R. Lacource, T. Morgues, and M. E. Goodrich. Growth rate of mycobacteria in mice as an unreliable indicator of mycobacterial virulence. Infect. Immun., 67:5483-5485, 1999.

[50] E. G. Hoal-nan Helden, D. Hon, L. A. Lewis, N. Beyers, and P. D. van Helden. Mycobacterial growth in human macrophages: variation according to donor, inoculum and bacterial strain. Cell Biol. Int., 25:71-81, 2001.

[51] O. J. Billington, T. D. Mchugh, and S. H. Gullespie. Physiological cost of rifampicin resistance induced in vitro in mycobacterium tuberculosis. Antimicrob. Agents Chemother., 43:1866-1869, 1999. 
[52] C. Dye and B. G. Williams. Criteria for the control of drug-resistant tuberculosis. Proc. Natl. Acad. Sci. USA, 97:8180-8185, 2000.

[53] C. Dye and M. A. Espinal. Will tuberculosis become resistant to all antibiotics? Proc. R. Soc. Lond. B, 268:45-52, 2001.

[54] A. Telenti and M. Iseman. Drug-resistance tuberculosis. what do we know? Drugs, 59:171-179, 2000. 